\title{
Die Weiterentwicklung der Universitäts-Hautklinik Kaunas/Litauen in enger Zusammenarbeit mit deutschsprachigen Dermatologen
}

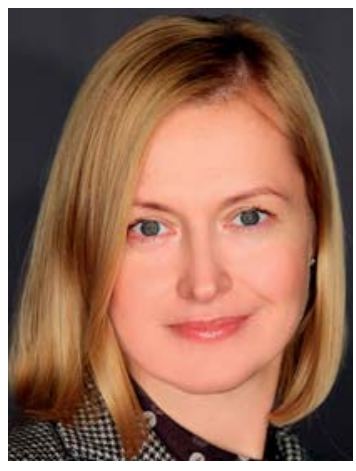

Prof. Skaidra Valiukeviciene
Mit großer Freude möchte ich Ihnen die Universitätsklinik für Haut- und Geschlechtskrankheiten Kaunas in dieser Ausgabe der Aktuellen Dermatologie vorstellen. Kaunas war etwa 20 Jahre vor dem Zweiten Weltkrieg die vorübergehende Hauptstadt Litauens. Aktuell ist Kaunas die zweitgrößte Stadt Litauens mit etwa vierhunderttausend Einwohnern. Bis zum heutigen Tag hat sich die Stadt die Rolle eines wichtigen Industrie-, Kultur- und Studentenzentrums erhalten. Im Jahr 1923 erhielt Kaunas den Status Litauen Vytautas Magnus Universität und die erste Universitätsklinik für Haut- und Geschlechtskrankheiten wurde errichtet. Vor dem Zweiten Weltkrieg waren in der Klinik die wichtigsten Gebiete der Forschung und der praktischen Tätigkeit die Venerologie, die Hautinfektionen und die Dermatoallergologie. Der Leiter der Universitäts-Hautklinik Kaunas, Bronius Sidaravičius (1897-1969), war sehr eng mit deutschsprachigen Dermatologen verbunden. Er hospitierte als Stipendiat an der Universität Wien in der Innsbrucker Klinik und forschte dort mit Erich Urbach (1893-1946) an der passiven Übertragung der Hautallergien. Seit der Gründung hat die Universitäts-Hautklinik Kaunas einen langen und schwierigen Weg der räumlichen Veränderungen und politischen Reformen überstanden, bis sie im Jahr 2001 wieder im Universitätsklinikum Kaunas eröffnet wurde. Zu der Zeit hat die Zusammenarbeit mit deutschsprachigen Dermatologen und die Zusammenarbeit auf einem interdisziplinären Kampus des Universitätsklinikums Kaunas den Aufbau der Universitäts-Hautklinik geprägt. So entwickelten sich neue Fachbereiche wie die interdisziplinäre Dermatoonkologie, Dermatochirurgie und Fotochemotherapie an der Universitäts-Hautklinik Kaunas. In letzten zehn Jahren hat sich die Zahl der Patienten in der Ambulanz verdoppelt und in den letzten 5 Jahren wurden teilstationäre Behandlungen eingeführt. Chronische entzündliche Dermatosen wie Psoriasis, Kontakt- und atopisches Ekzem sind führende Diagnosen auf der 16-Betten-Station. Doch in der Ambulanz vertei- len sich die häufigsten Diagnosen auf Hauttumoren, Psoriasis und Kontaktekzem. Die Forschungstätigkeiten der Universitäts-Hautklinik Kaunas umfassen epidemiologische und klinische Studien zu Hauttumoren, Akne, Kontaktekzem und Geschlechtskrankheiten. Zwei Eurostar- und ein Framework-7-Projekt (2009-2014) schließen die Etablierung der innovativen bildgebenden Ultraschall-Technologien ein in einer Kombination mit der Thermografie, Dermatoskopie und Spektrofotometrie für die Verbesserung der Frühdiagnostik des diabetischen Fußes und des malignen Melanoms der Haut. Bei der Durchführung der Forschungsprojekte arbeitet die Klinik eng zusammen mit dem Ultraschall-Institut der Technischen Universität Kaunas, dem ESSCA (European Surveillance System on Contact Allergy), dem CVDerm Zentrum (Hamburg), der Otto-von-Guericke Universität Magdeburg und der Universität Halle. Eine wichtige Tätigkeit der Klinik ist die Ausbildung der Studenten und Fachärzte. Seit dem Jahr 2005 läuft das von 2 auf 4 Jahre verlängerte Curriculum zur Facharztausbildung in der Dermatovenerologie. Das Pre-Diplom-Studium in der Dermatovenerologie umfasste in Zeit und Studenten im Jahr 2013 jeweils 60 Kontaktstunden der 350 Medizinstudenten, 30 Kontaktstunden der 142 Odontologen, 40 Kontaktstunden der 56 Nurses und 10 Kontaktstunden der 21 Public Health-Studenten.

Die stetig wachsende Anzahl der dermatologischen Patienten und Studenten zeigt, dass die Universitäts-Hautklinik Kaunas den Status eines wichtigen regionalen Zentrums der dermatologischen und venerologischen Hilfe bis zum heutigen Tag erhalten und weiter vertieft hat. Die litauischen Dermatologen sind den deutschsprachigen Kollegen sehr dankbar für die ständige Unterstützung des Faches und bedanken sich für die Einladung, die Universitäts-Hautklinik Kaunas in dieser Ausgabe der Aktuellen Dermatologie vorzustellen.

Prof. Skaidra Valiukeviciene 